\title{
Efficacy of traditional Chinese medicine in treating cancer (Review)
}

\author{
JIAO NIE $^{1 *}$, CHANGLIN ZHAO $^{1,2^{*}}$, LI DENG $^{1}$, JIA CHEN $^{1}$, BIN YU $^{1,3}$, \\ XIANLIN WU ${ }^{1,4}$, PENG PANG ${ }^{1}$ and XIAOYIN CHEN ${ }^{1}$ \\ ${ }^{1}$ Department of Traditional Chinese Medicine, Medical College of Jinan University; ${ }^{2}$ Department of Oncology, \\ Clifford Hospital of Guangzhou University of Traditional Chinese Medicine, Guangzhou, Guangdong 510632; \\ ${ }^{3}$ Clinical Institute, Jining Medical University, Jining, Shandong 272013; ${ }^{4}$ Department of Pancreatic Disease Center, \\ The First Affiliated Hospital of Jinan University, Guangzhou, Guangdong 510632, P.R. China
}

Received August 18, 2015; Accepted September 25, 2015

DOI: $10.3892 /$ br.2015.537

\begin{abstract}
The morbidity associated with cancer has rapidly increased in recent years, and in the previous 5 years has had a tendency to be the leading cause of fatality compared with cardiovascular disease. Therefore, effective measures are required with an aim to reduce the incidence. Based on the results of clinical investigation, a multidisciplinary treatment strategy for cancer, which includes radiotherapy, chemotherapy, surgery, targeted therapy and immunotherapy, are prominently used in clinical practice. However, the therapies are insufficient due to multidrug resistance, adverse effects and the presence of the root of the cancer. Therefore, there is a necessity to develop more effective or adjunctive therapies for cancer prevention and treatment. Cancer is now widely recognized as a systemic humoral disease. Similarly, the function of herbal drugs is to modulate the whole body system in a more holistic way. Recently, herbal drugs have been applied to one of the efficient approaches for cancer therapy. Furthermore, there is evidence that various herbal medicines have been proven to be useful and effective in sensitizing the conventional agents against the various factors at the cellular and molecular levels that are associated with the occurrence of cancer and in prolonging survival time, alleviating side effects of chemotherapy and radiotherapy and improving the quality of life in cancer patients.
\end{abstract}

Correspondence to: Professor Xiaoyin Chen, Department of Traditional Chinese Medicine, Medical College of Jinan University, 601 Huangpu Road West, Guangzhou, Guangdong 510632, P.R. China E-mail: tchenxiaoyin@jnu.edu.cn

*Contributed equally

Key words: cancer, herbal medicines, cellular and molecular level, survival time, side effects, quality of life

\section{Contents}

1. Introduction

2. Inducing cellular apoptosis

3. Reversing multidrug resistance

4. Comprehensive efficacy on angiogenesis, cellular signaling pathway and metastasis

5. Regulating the immunomodulatory activities

6. Enhancing the efficacy, reducing toxicity and improving the quality of life for patients

7. Conclusion and future perspectives

\section{Introduction}

The database from the American Cancer Society (ACS) provides a summary of the current ACS cancer screening rates. Aside from skin cancer, the major incidence of cancer in 2015 was reported as cancer of the female breast, prostate cancers in males, and colorectal and lung cancer (1), and according to the study of Bray et al (2) reported in Lancet Oncology, the incidence of the total cancer cases will increase from 12.7 million new cases in 2008 to 22.2 million by 2030 . Therefore, cancer specialists have determined that cancer is a life-threatening disease for future generations (3). Therefore, it is necessary to implement national cancer control plans to reduce the burden of cancer risk factors. Increasing attention has been paid to improve the palliative care and pain relief available to patients. Even though the skills of surgery, radiotherapy and chemotherapy, targeted therapy and immunotherapy have significantly improved, an increasing number of patients are selecting naturopathic therapy with traditional Chinese medicine (TCM), as they are considered to be multicomponents and multitarget agents exerting their therapeutic function in a more holistic way to the whole body system via restoration of the normal balance and flow in the body, which is believed to strengthen and enhance the endogenous resistance of the body to disease and individualization of therapy. In addition, the approval of three new herbal medicine-derived drugs (ixabepilone, trabectedin and temsirolimus) (4) in 2007 instigated the consideration of herbal medicines as a source for innovative 
antitumor-targeted therapeutic agents. Worldwide, including Western countries, TCM has been more accepted for its efficacy in preventing and treating cancer. Investigations of targeted therapy with herbal medicines will potentially provide future discoveries in oncological research. It is estimated that the United States National Cancer Institute spends 120 million USD each year on TCM-related research (5). While, based on the foundation of the system research of 'omics' approach (6), the occurrence of cancer is known to be based on various factors, several phases, multigenes and complicated signaling pathways $(7,8)$. Cancer is considered a chronic health problem, similar to hypertension and diabetes by the World Health Organization, and therefore, the optimal effects of treatment involve improving life quality, prolonging survival time and alleviating side effects. Therefore, the concept of 'survival with cancer' has been suggested (9). The following is the evidence reported in preclinical or clinical trials that demonstrates the mechanism and efficacy of medicinal plants used as adjuvant treatment for cancer patients (also shown in Table I).

\section{Inducing cellular apoptosis}

Cell apoptosis is a form of programmed cell death that induces changes in cellular morphology and intracellular processes without inflammatory reactions. The procedures of apoptosis are formed as follows: Receiving apoptotic signals leads to cytomorphological alterations, the interaction between the intermolecular apoptotic proteins, activation of the caspase enzyme that acts upon cytoskeleton proteins, mitochondria, nuclear membrane or chromatin to cleave proteins and substrates, causing the DNA to fragment, releasing the cytoplasm enclosed by the plasma membrane, eventually leading to cell death (10-14). Currently, it is known that the extrinsic or death receptor pathway, the intrinsic or mitochondrial pathway and the perforin/granzyme pathway are the major apoptotic pathways to activate caspases $(12,15-17)$. The apoptotic process is involved in the maintenance of tissue homeostasis, which can be triggered by a variety of stimuli, including cytokines, hormones, toxic insults and viruses. Once these stimuli seriously affect the self-homeostasis of the cells, the genes, including oncogenes, tumor-suppressor genes, suicide genes and DNA-repair genes, are activated and become cancerous resulting in the continuous division of the faulty cells. However, the herbal medicines have an important role in the prevention and treatment of cancer and execute their multiple therapeutic effects by inhibiting cancer-activating enzymes, stimulating a DNA repair mechanism and promoting production of protective enzymes, thus, showing anticancer effects.

Lin et al (18) investigated the cellular effects of the ethane extract of Hedyotis Diffusa Willd. (EEHDW) in the HT-29 human colon carcinoma cell. The study reported that EEHDW inhibited the growth of HT-29 cells by inducing cell morphological changes, which reduce the cell viability due to DNA fragmentation and loss of plasma membrane asymmetry, and also the collapse of mitochondrial membranes, the activation of caspase- 9 and caspase- 3 and the increase of the pro-apoptotic Bax to anti-apoptotic Bcl-2 ratio. Oxymatrine (19) is an isolated extract from the traditional Chinese herb Sephora flavescent Ait, and its anticancer effect was examined on human pancreatic cancer PANC-1 cells. The results showed its efficacy by releasing cytochrome $c$ and activating caspase-3 proteins. Additionally, it downregulated the expression of living and surviving genes and the $\mathrm{Bax} / \mathrm{Bcl}-2$ ratio was unregulated. The study by Guo et al (20) demonstrated that oxymatrine effectively suppressed the cell proliferation and phosphorylation of EGFR (Tyr845), decreased phospho-cofilin (Ser3) and phospho-LIMK1 (Thr508) without changing the total cofilin and LIMK1 expression to inhibit the migration and invasion of human gastric cancer cells. Hu et al (21) studied the efficacy of the TCM tubeimu on the MDA-MB-453 human breast cancer cell line. The study reported that the fragmentation of DNA could be observed by a fluorescence microscope at $72 \mathrm{~h}$ and apoptosis frequently increased when the cells were in a three-dimensional compared to a two-dimensional culture. The research regarding the emodin azide methyl anthraquinone derivative (22) demonstrates that cytotoxic effects (such as cell death) are through the arrest of the cell cycle and the induction of apoptosis in cancer cells. The overall molecular mechanisms of emodin include cell cycle arrest and apoptosis, promote the expression of hypoxia-inducible factor $1 \alpha$, glutathione S-transferase $\mathrm{P}$ and $\mathrm{N}$-acetyltransferase, and induce glutathione phase I and II detoxification enzymes to inhibit angiogenesis, invasion, migration and chemical-induced carcinogen-DNA adduct formation. HER2/neu, CK2 kinase and p34cdc2 kinase were also inhibited in the human breast cancer cells.

\section{Reversing multidrug resistance}

Multidrug resistance (MDR) represents a prominent obstacle in cancer chemotherapy. Therefore, with the elucidation of the MDR mechanism, MDR has been associated with an adenosine triphosphate (ATP)-dependent decrease in cellular drug accumulation, which is attributed to the overexpression of certain ATP-binding cassette (ABC) transporter proteins (23). There are 9 human MDR proteins that are generally members of subfamily $\mathrm{C}$ in the ABC superfamily (23). Although their structures are different, they share a similar ATP-driven transport mechanism (24). However, due to their different locations, expression levels and activities, the drug resistance to chemotherapy is varied. Using the method for identifying the MDR mechanisms, there are three major mechanisms of drug resistance known in cells: i) Decreased water-soluble drug absorption, including folate antagonists and nucleoside analogues, which require transporters to enter cells; ii) various changes in cells occur that affect the capacity of cytotoxic drugs to kill cells, such as alterations in cell cycle, increased repair of DNA damage, reduced apoptosis and altered metabolism of drugs; and iii) increased energy-dependent efflux of hydrophobic drugs that can easily enter the cells by diffusion through the plasma membrane (25). P-glycoprotein (P-gp), which belongs to the first identified member of the ABC transporter superfamily of membrane transport proteins (26), represents the most common capability of resistance to hydrophobic anticancer drugs by actively extruding the drugs from the cells, including vinblastine, daunorubicin and paclitaxel. Therefore, patients with MDR tumor types often only have limited options, but can withstand significantly high doses (27). Thus, with the evidence that more new drugs are deriving from herbal medicines, quercetin, epigallocatechin gallate, curcumin, capsaicin and gingerol have 


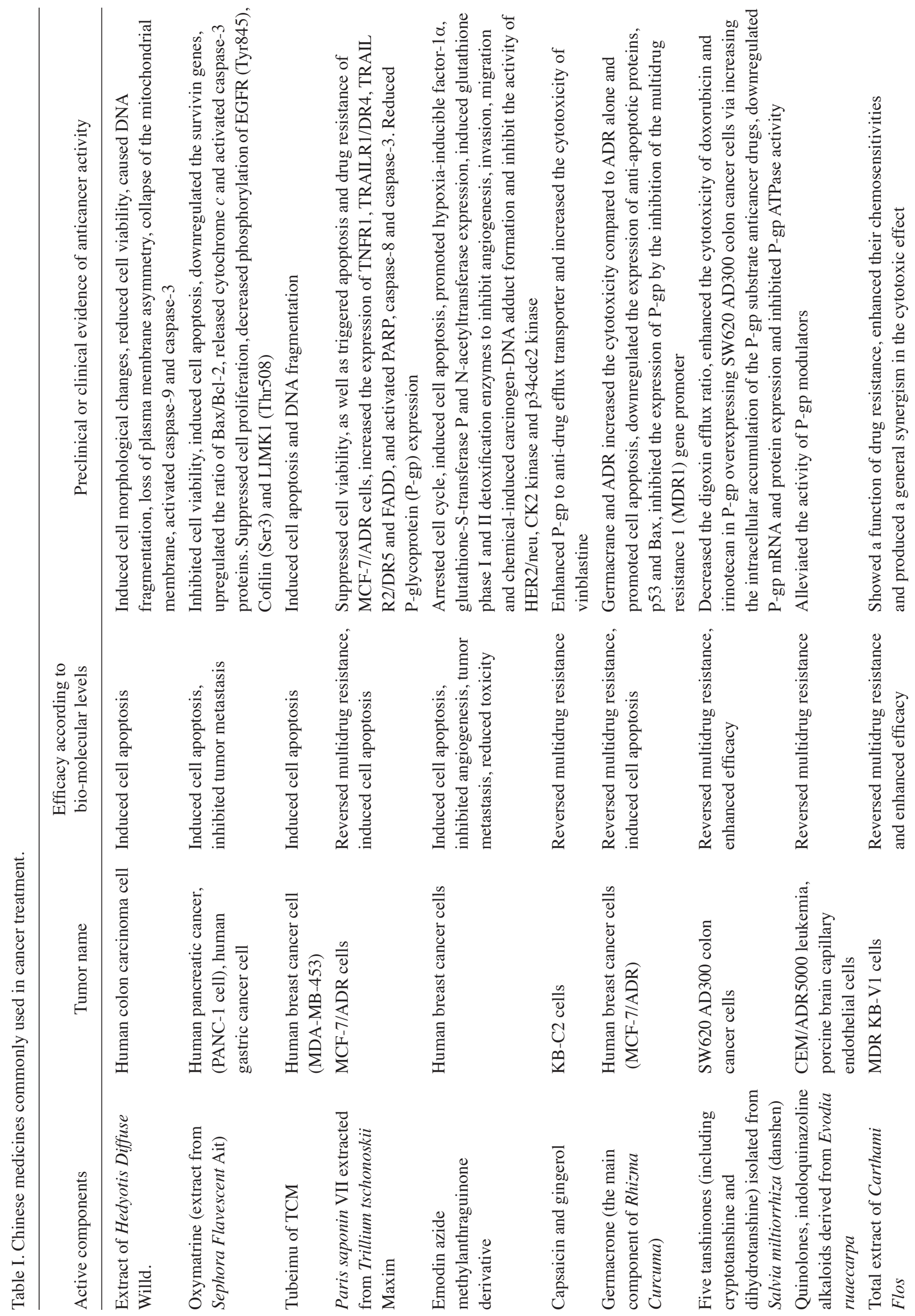




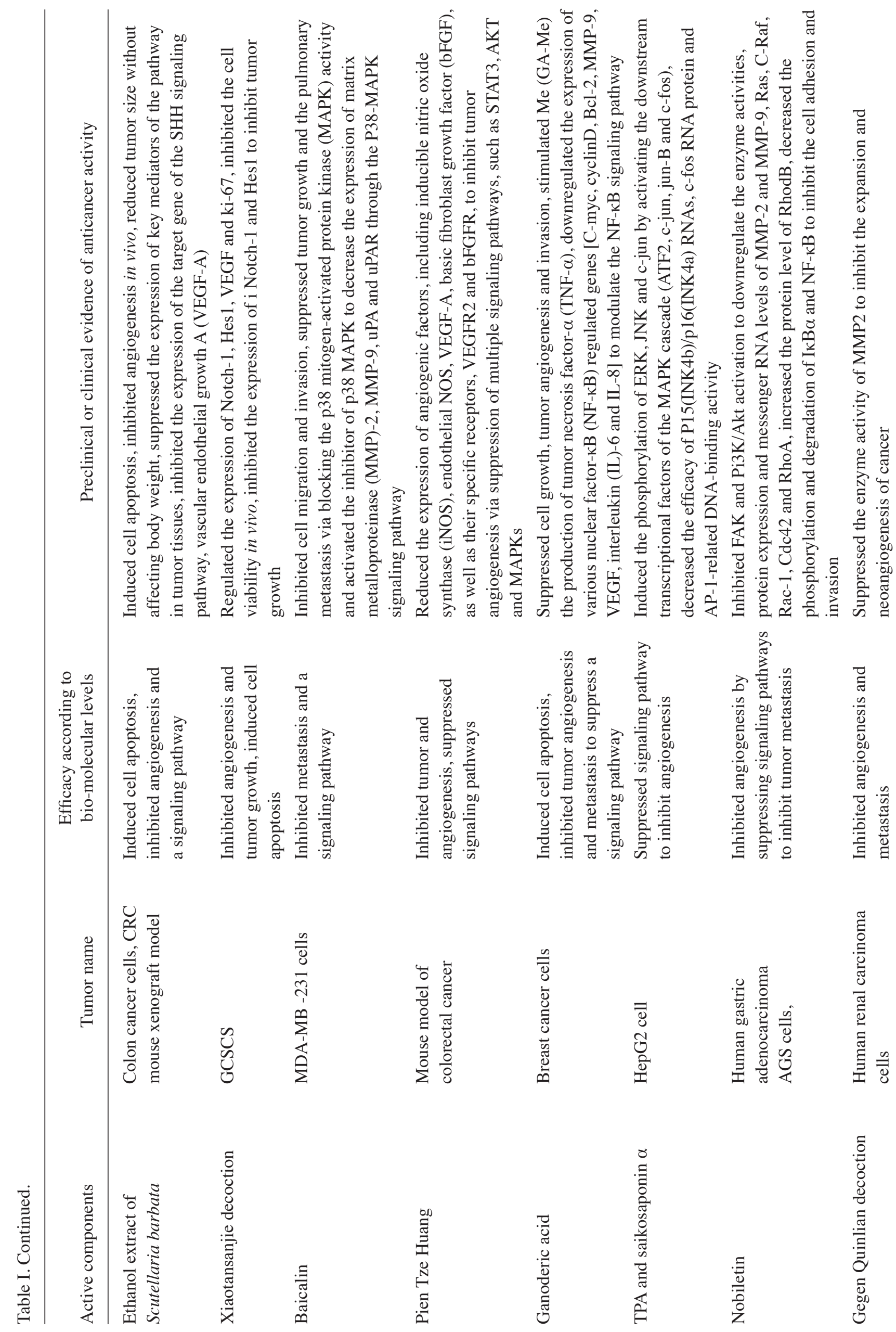




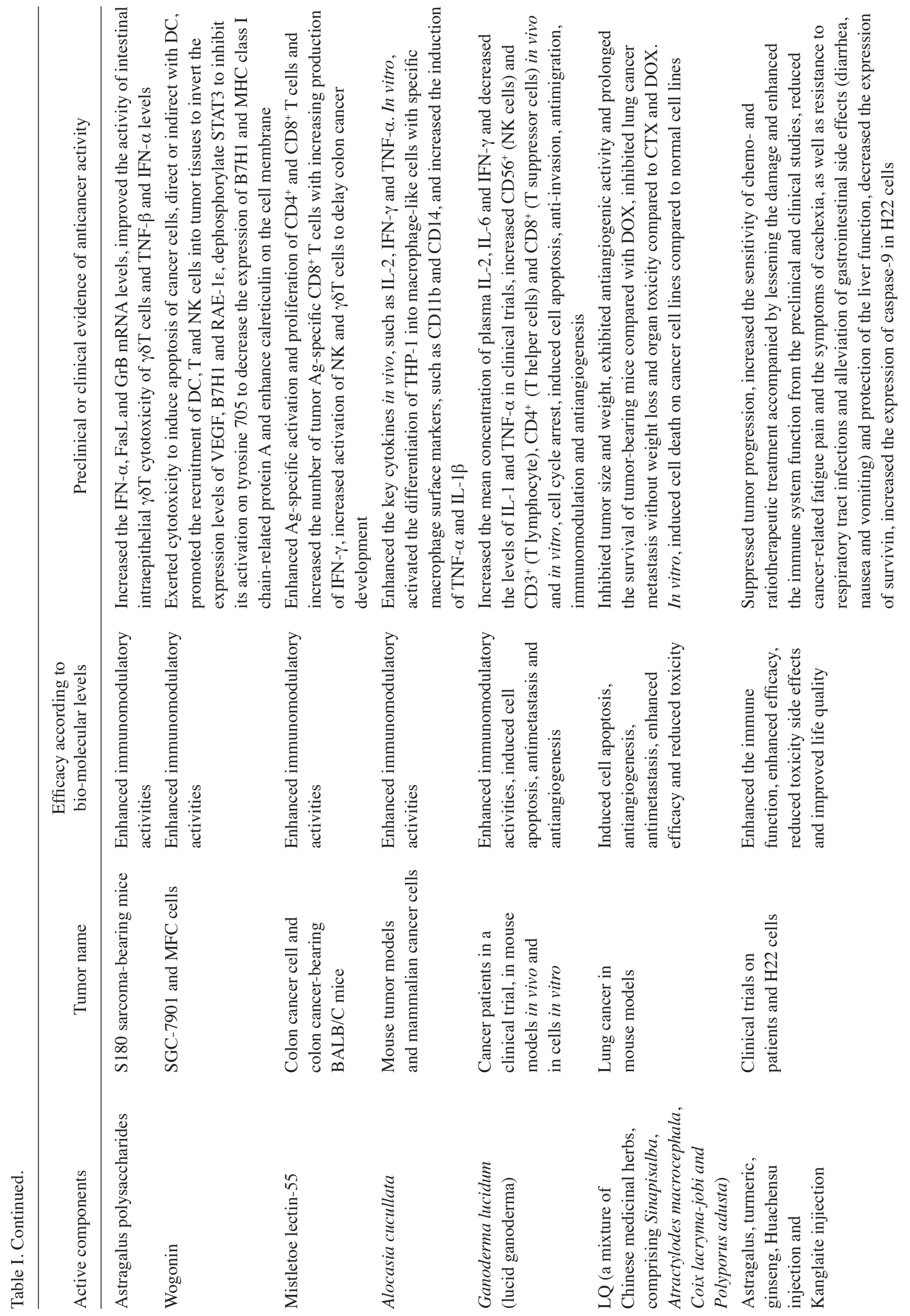




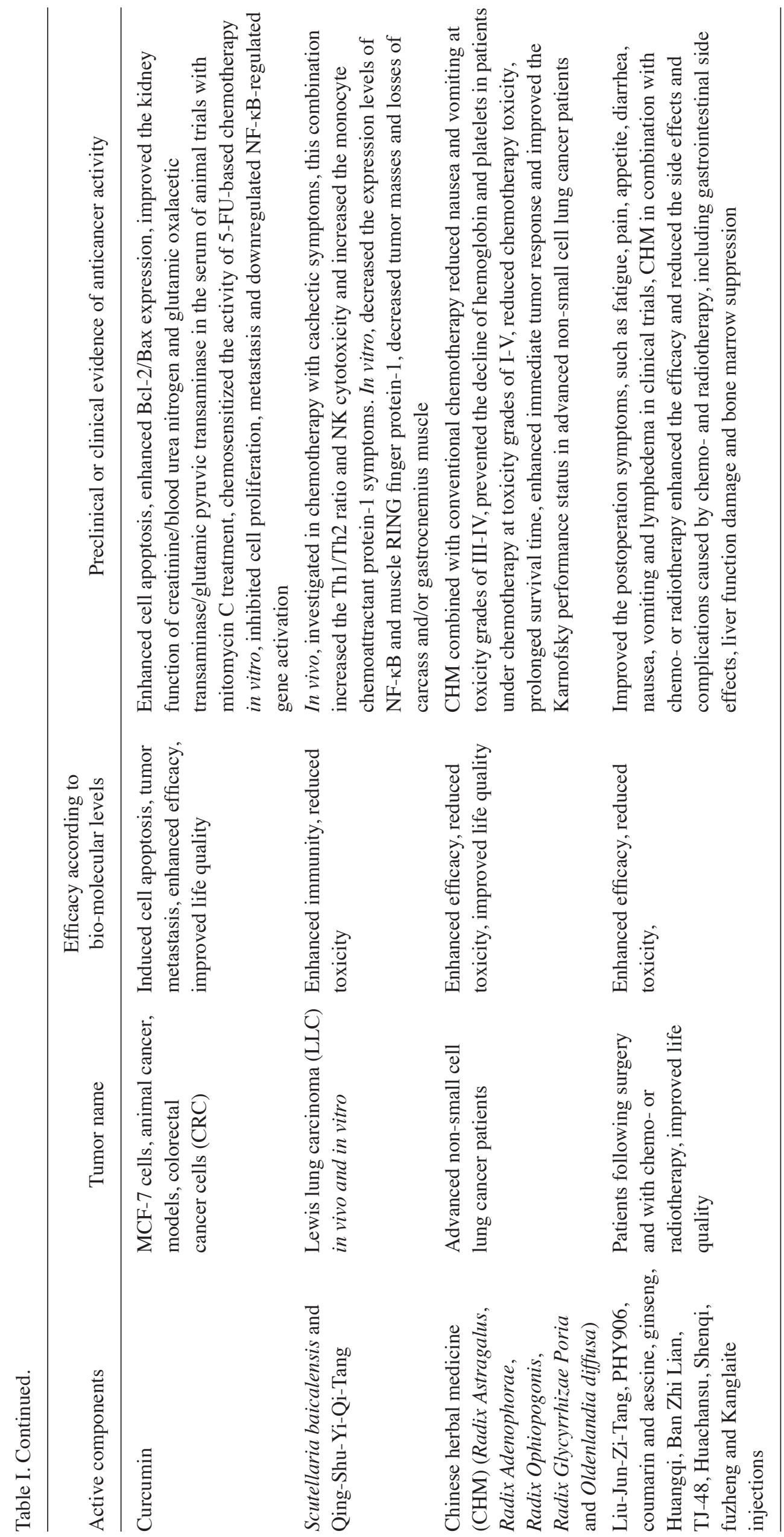


become promising candidates (28) as they overcome MDR potency in antitumor treatment. They act as MDR inhibitors, MDR modulators, MDR reversal agents or chemosensitizers to reverse the resistance against anticancer drug (25-30). Therefore, increasing studies are focusing on exploiting MDR reversal compounds from medicinal plants, as they have low or no side effects for use in cancer treatment (29), to bypass drug resistance in treating cancer, and eventually medicinal herbs may be applied in the future in cancer adjuvant therapy.

Capsaicin (8) and gingerol (8) have been reported to exhibit inhibitory effects on human P-gp. They increased the intracellular concentration of P-gp substrates by inhibiting this anticancer drug efflux transporter. MDR carcinoma KB-C2 cells were more susceptible to the cytotoxicity of vinblastine, as compared with vinblastine alone, when simultaneously treated with $50 \mu \mathrm{M}$ capsaicin or gingerol (31). Therefore, capsaicin and gingerol can partially reverse MDR in cells that express P-gp. Germacrone (32), the main component of Rhizma curcuma, was tested on MCF-7/adriamycin (ADR) MDR human breast cancer cells. The combination of germacrane and ADR increased the cytotoxicity when compared with ADR alone, and promoted cell apoptosis in a dose-dependent manner. The apoptotic effect was enhanced with ADR, as analyzed by downregulating the expression of anti-apoptotic proteins p53 and Bax. In addition, germacrone inhibited the expression of P-gp by inhibiting the MDR1 gene promoter. Tao et al (33) investigated the reversal of P-gp in colon cancer cells by five tanshinones isolated from Salvia miltiorrhiza (danshen), the result demonstrated that only cryptotanshinone and dihydrotanshinone decreased the digoxin efflux ratio in a dose-dependent manner and they enhanced the cytotoxicity of doxorubicin and irinotecan in P-gp overexpressing SW620 AD300 colon cancer cells by increasing the intracellular accumulation of the P-gp substrate anticancer drugs, which possibly downregulate the P-gp mRNA and protein expression and inhibit P-gp ATPase activity. Paris saponin VII (27), a type of saponin extracted from Trillium tschonoskii Maxim, dose-dependently suppressed cell viability, as well as triggered apoptosis and drug resistance of MCF-7/ADR cells. Furthermore, it also increased the expression of TNFR1, TRAILR1/DR4, TRAILR2/DR5 and FADD, and activated PARP, caspase- 8 and caspase- 3 . By contrast, the P-gp expression and activity were reduced. Quinolones, indoloquinazoline alkaloids that are derived from the plant medicine Evodia ruaecarpa, were tested on CEM/ADR5000 leukemia and porcine brain capillary endothelial cells. The results showed that the quinolones are moderate modulators of P-gp activity $(34,35)$. Additionally, the drug resistance index of the total extract of Carthami Flos (CF) in MDR KB-V1 cells and its synergistic effects with other chemotherapeutic agents were studied. The results revealed that $\mathrm{CF}$ showed a drug resistance index, according to the classic isobologram equation. However, in combination with other chemotherapeutic agents, it enhanced their chemosensitivities and provided a general synergism of the cytotoxic effect (36).

\section{Comprehensive efficacy on angiogenesis, cellular signal- ing pathway and metastasis}

Angiogenesis is the formation of new blood vessels and is a complex and regulated process that involves a number of intercellular pro- and antiangiogenic signaling pathways. These pathways control the activity of blood vessel-associated cells, including endothelial cells (ECs) and pericytes (37), which is formed by the initiation of cell proliferation and migration in response to angiogenic stimulators: Vascular endothelial grow th factor (VEGF), fibroblast growth factor-2 (FGF-2), interleukin-8 (IL-8), placental growth factor (PlGF), transforming growth factor- $\beta$ (TGF- $\beta$ ), platelet-derived growth factor (PDGF), nitric oxide (NO), ephrins, angiopoietins, endothelins, integrins, cadherins, chemokines and Notch (37-39). The crosstalk among the overexpression of growth factors, hypoxia-inducible factor and the dysregulation of tyrosine kinase receptors is controlled by the phosphoinositide-3-kinase (PI3K)/Akt/mTOR (40), Ras/Raf/ERK/mitogen-activated protein kinase (MAPK) and hypoxia-inducible factor (HIF) regulatory pathways $(41,42)$. The complicated formation of angiogenesis has provided a platform for tumor metastasis. The tumor hypoxia-induced epithelial-to-mesenchymal transition (EMT) also increased the metastatic phenotype of tumors (43). When oxygen and nutrients are supplied to the tumor cells, angiogenesis has an important role in the growth, invasion and metastasis for the tumor. Therefore, when the tumor microenvironment has been converted by angiogenesis, antiangiogenesis becomes a challenge for antitumor treatment. Therefore, numerous studies have inhibited tumor angiogenesis from four aspects $(37,38,42)$ : One is restraining the inhibitor of tumor angiogenesis degraded by the basilar membrane, and the second is inhibiting the inhibitor of tumor angiogenesis for increasing endotheliocytes. The third is preventing the activation of the growth factor that is associated with tumor angiogenesis. The final aspect is inhibiting the signaling pathways associated with tumor angiogenesis. However, the efficacy is unilateral, which may be insufficient and possibly generate drug resistance (44). Therefore, these problems highlighted the necessity for the exploitation of novel anticancer agents. However, there is numerous evidence (41) that has proved that plant-derived medicinal compounds may, in a number of cases, be at least as effective in blocking angiogenesis as the currently used synthetic drugs, which exhibit only a fraction of the side effects. The following are examples of the plant-derived medicinal compounds.

The TCM Scutellaria barbata is commonly used in cancer treatment. The ethanol extract of Scutellaria barbata (45) was able to induce apoptosis of the colon cancer cells and inhibit angiogenesis in a chick embryo chorioallantoxic membrane model. In a colorectal cancer cell (CRC) mouse xenograft model in vivo, it could reduce tumor size without affecting the body weight and suppress the expression of key mediators of the Sonic hedgehog (SHH) pathway in tumor tissues. The expression of the important target gene of the SHH signaling pathway, vascular endothelial growth factor A (VEGF-A), was also inhibited, which has an important role in stimulating tumor angiogenesis. The study of the xiaotansanjie decoction (46) in regulating the expression of Notch-1, Hes1, VEGF and Ki-67 was measured by western blotting and reverse transcription-polymerase chain reaction and the cell viability was measured by the MTT assay. The data revealed that cluster of differentiation (CD) $44^{+}$gastric cancer stem cells (GCSCS) showed more cell proliferation and VEGF secretion compared to CD44- cells in vitro, 
whereas in vivo, the CD $44^{+}$GCSCS had a high expression of Notch-1 and Hes1, which was positively associated with tumor growth. The study by Wang et al (47) reported that baicalin not only dose-dependently inhibited MDA-MB-231 cell migration and in vitro invasion, but also suppressed the growth of the tumor and the pulmonary metastasis via blocking p38 MAPK activity and activated the inhibitor of p38 MAPK which decreased the expression of matrix metalloproteinase-2 (MMP-2), MMP-9, urokinase plasminogen activator (uPA) and uPA receptor through the p38-MAPK signaling pathway. Pien Tze Huang (48) was tested on a mouse model of colorectal cancer and the treatment reduced the expression of angiogenic factors, including inducible NO synthase (NOS), endothelial NOS, VEGF-A, basic FGF (bFGF), as well as their specific receptors VEGFR2 and bFGFR, to inhibit the tumor angiogenesis via suppressing multiple signaling pathways, such as STAT3, AKT and MAPKs. In addition, based on the investigation of ganoderic acid $\mathrm{Me}$ (GA-Me) (49), the growth, angiogenesis and invasion of breast cancer cells were suppressed. By contrast, tumor necrosis factor- $\alpha(\mathrm{TNF}-\alpha)$ was stimulated. Furthermore, GA-Me downregulated the expression of various nuclear factor- $\kappa \mathrm{B}(\mathrm{NF}-\kappa \mathrm{B})$-regulated genes (C-myc, cyclin D, BCL-2, MMP-9, VEGF, IL-6 and IL-8) to modulate the NF- $\kappa$ B signaling pathway. Saikosaponin (50) was investigated on the HepG2 cell line and the results revealed that phosphorylation of ERK was significantly induced by TPA and saikosaponin $\alpha$, whereas the phosphorylation of JNK was induced only by TPA and the phosphorylation of P38 was not induced by either. This reaction was derived from the activation of the downstream transcriptional factors of the MAPK cascade (ATF2, c-jun, jun-B and c-fos). However, the inhibitor could decrease the efficacy of p15 (INK4b)/p16 (INK4a) RNAs, c-fos RNA protein and AP-1-related DNA-binding activity by TPA and saikosapanin, and c-jun phosphorylation was only reduced by TPA. A study of nobiletin (51) treatment of human gastric adenocarcinoma AGS cells reported an inhibition of the activation of focal adhesion kinase and PI3K/Akt, which are involved in the downregulation of the enzyme activities, and protein and messenger RNA levels of MMP-2, MMP-9, Ras, c-Raf, Rac-1, Cdc42 and RhoA. The protein level of RhoB was progressively increased, whereas treatment decreased the phosphorylation and degradation of inhibitor of $\kappa \mathrm{Ba}(\mathrm{I} \kappa \mathrm{Ba})$ and the nuclear response element of NF- $\kappa \mathrm{B}$ also exerted inhibitory effects on the cell adhesion and invasion. The TCM Gegen Qinlian decoction (52) significantly suppressed the enzyme activity of MMP-2 in human renal carcinoma cells to inhibit the expansion and neoangiogenesis of cancer.

\section{Regulating the immunomodulatory activities}

To an extent, tumors produce a number of tumor antigens, which are the primary triggers of the immune response. They are activated via a major histocompatibility complex (MHC) and the $\mathrm{T}$ cell response. In the process of immunosurveillance, they trigger T-cell activation to recruit other immune effector cells with the expression of co-stimulatory molecules and secretion of chemokines and cytokines to regulate different aspects of the immune response, including Th1 CD4 T cells that activate CD8 $\mathrm{T}$ cells, and subsequently CD8 T cells are activated by direct antigen presentation, via MHC class I or via CD4 T-cell-mediated activation. The tumor cell is ultimately destroyed by direct cell-mediated cytotoxicity, as well as an indirect antibody complement-mediated cytotoxicity (53). However, the majority of tumors have been demonstrated to lose the expression of MHC molecules, resulting in the loss of presenting tumor antigens, thus evading T-cell recognition $(40,53)$. In addition, when the balance of the immune checkpoint was destroyed, certain tumors upregulated inhibitory molecules to evade the immune response (40). Due to the strong heterogeneity of cancer, exploring novel effective drug targets and therapeutic strategies to overcome the problem is required. TCM, with a long history of treating various diseases through modulating the human immune response, offers a potential to be a remedy of the new immunomodulating regimen for cancer treatment. They exert broad immunostimulatory activities by recruiting and activating different types of immune cells, such as dendritic cells (DC), natural killer (NK) T-cells, T cells, macrophage, monocytes (54-56) and adjusting the production of cytokines (IFN- $\alpha$, IL-2, IL-6 and IFN- $\gamma)(54,57,58)$ to fight against the tumor cells.

In the study of astragalus polysaccharides (59) conducted on S180 sarcoma-bearing mice, the IFN- $\alpha$ FasL and GrB mRNA levels were increased and the activity of intestinal intraepithelial $\gamma \delta \mathrm{T}$ cells in vivo was also improved. The cytokine production and cytotoxicity of $\gamma \delta \mathrm{T}$ cells and the levels of TNF- $\beta$ and IFN- $\alpha$ were all markedly improved following treatment. The study of wogonin (54) treatment revealed that it exerted cytotoxicity, directly or indirectly with DC, to induce apoptosis of cancer cells (SGC-7901 and MFC cells). The treatment also promoted the recruitment of $\mathrm{DC}, \mathrm{T}$ and NK cells into tumor tissues to invert the expression levels of vascular endothelial growth factor, B7H1, and RAE-1ع in tumor tissues. Additionally, the treatment also dephosphorylated STAT3 to inhibit its activation on tyrosine 705 in tumor cells, which contributed to a decrease in the expression of $\mathrm{B} 7 \mathrm{H} 1$ and $\mathrm{MHC}$ class I chain-related protein $\mathrm{A}$ and the enhancement of calreticulin on the cell membrane. Notably, these data indicated that wogonin could be used in collaboration with a DC vaccine or activated lymphocytes for tumor therapy. In addition, active chinese mistletoe lectin-55 was tested on colon cancer, and the results demonstrated that it enhanced antigen (Ag)-specific activation and proliferation of $\mathrm{CD}^{+}$and $\mathrm{CD} 8^{+} \mathrm{T}$ cells and increased the number of tumor Ag-specific $\mathrm{CD} 8^{+} \mathrm{T}$ cells with increasing production of IFN- $\gamma$. Of note, the treatment with ACML-55 (58) also showed increased activation of innate lymphocytes, such as NK and $\gamma \delta \mathrm{T}$ cells. These immune responses significantly delayed colon cancer development in colon cancer-bearing BALB/C mice. Alocasia cucullata (AC) (57), a Chinese medicine, was investigated on mouse tumor models cultured in mammalian cells, and the results showed that its antitumor effect was closely associated with a strong induction of key cytokines in vivo, such as IL-2, IFN- $\gamma$ and TNF- $\alpha$. AC activated the differentiation of THP-1, a human monocytic cell line, into macrophage-like cells in vitro, accompanied with specific macrophage surface markers, such as CD11b and CD14, and increased 
the induction of TNF- $\alpha$ and IL-1 $\beta$. All these data verified the potential of AC to be an alternative immunomodulating herbal remedy for anticancer treatment. Ganoderma lucidum (lucid ganoderma) (60), a traditional Chinese herb, has been used extensively in East Asia for one thousand years. Compounds of the extraction of Ganoderma lucidum mixed in PC-SPES $(61,62)$ were administered to cancer patients in a clinical trial, in mouse models in vivo and in cells in vitro. In the prostate cancer patients, it improved the immunostimulating response with an increased concentration of plasma IL-2, IL- 6 and interferon- $\gamma$, and it enhanced the natural killer cell activity and decreased the production of plasma IL-1 and tumor necrosis factor- $\alpha$. Simultaneously, in patients with advanced-stage cancer in a clinical trial, the statistical results showed that following surgery, the patients undergoing oral administration of lucid ganoderma extract for 12 weeks exhibited a significantly increased mean concentration of plasma IL-2, IL- 6 and IFN- $\gamma$, and significantly decreased levels of IL- 1 and TNF- $\alpha$. There was also a significant increase of $\mathrm{CD}^{2} 6^{+}(\mathrm{NK}$ cells) and a small increase of $\mathrm{CD}^{+}$( $\mathrm{T}$ lymphocyte), $\mathrm{CD}^{+}$(T-helper cells) and $\mathrm{CD}^{+}$(T-suppressor cells) with unchanged CD4:CD8 T-cell ratios, which were induced by the lucid ganoderma extract as compared to the baseline levels (63). With regards to the in vitro and in vivo studies, the anticancer mechanism of lucid ganoderma treatment against the growth of different cancer cells appeared to be mediated by cell cycle arrest, apoptosis, anti-invasion, antimigration, immunomodulation and antiangiogenesis (60). This evidence greatly supported the anticancer potential of lucid ganoderma.

\section{Enhancing the efficacy, reducing toxicity and improving the quality of life for patients}

Over a few decades, systemic chemotherapy and radiotherapy have been the optimal treatment options for the tumor patients owing to the limitations and drawbacks (9) of surgery, including patients that are diagnosed too late to undergo surgery and a number of complications occurring following surgery. However, the major problem in cancer chemoradiotherapy is the prolonged toxicity of the well-established chemical drugs or the radioactive rays that resulted in numerous side effects and complications, including pain, diarrhea, nausea, vomiting, hair loss and hepatotoxicity. Even in advanced stages, certain patients show a syndrome of cachexia (64), which leads to a poor quality of life. Thus, the complementary and alternative medicines have become a preference for patients. Consequently, there is certain evidence in pre-clinical and clinical trials that has proved that TCM can enhance the efficacy and reduce toxicity with chemoradiotherapy and improve the quality of life and functional status of patients.

The study by Zhang et al (65) compared the efficacy of the TCM herbal mixture LQ (66) (a mixture of Chinese medicinal herbs, comprising Sinapis alba, Atractylodes macrocephala, Coix lacryma-jobi and Polyporus adusta) against lung cancer in mouse models using doxorubicin (DOX) and cyclophosphamide (CTX). LQ inhibited the tumor size and weight measured directly, as well as by fluorescent-protein imaging in subcutaneous and orthotropic experimental metastasis and angiogenesis lung cancer mouse models. Spontaneously, LQ had antiangiogenic activity and prolonged the survival of tumor-bearing mice compared with DOX. LQ was efficacious against primary and metastatic lung cancer without weight loss and organ toxicity, whereas CTX and DOX had opposing outcomes. In vitro, the study proved that LQ had the potential to induce cell death on cancer cell lines compared to normal cell lines. With regards to a review of Chinese herbal medicines (CHMs) (67) (astragalus, turmeric, ginseng, Huachansu injection and Kanglaite injection), data revealed that they have significant advantages in suppressing tumor progression, increasing the sensitivity of chemo- and ratiotherapeutics accompanied by lessening the damage of the treatment and enhancing the immune system function of the organism from the preclinical and clinical studies. Additionally, they reduce cancer-related fatigue and pain and the symptoms of cachexia, as well as resistance to respiratory tract infections, alleviation of gastrointestinal side effects (diarrhea, nausea and vomiting) and protection of the liver function. The expression of survivin was also decreased. However, the expression of caspase-9 increased in $\mathrm{H} 22$ cells. Furthermore, in the study of Zhou et al (68), mitomycin C (MMC) and curcumin synergistically enhanced apoptosis in MCF-7 cells by activating the caspases and enhancing the expression of $\mathrm{Bcl}-2 / \mathrm{Bax}$. Additionally, curcumin significantly improved the kidney function of creatinine/blood urea nitrogen and glutamic oxalacetic transaminase/glutamic pyruvic transaminase in the serum of animals undergoing MMC treatment. Furthermore, the study by Shakibaei et al (69) demonstrated that curcumin chemosensitized the activity of 5-FU-based chemotherapy on CRC accompanied with inhibition of cell proliferation, metastasis and downregulation of NF- $\mathrm{KB}$-regulated gene activation. Scutellaria baicalensis (70) and Qing-Shu-Yi-Qi-Tang (70) were investigated on tumor-bearing mice with cachectic symptoms undergoing chemotherapy, and this combination increased the Th1/Th2 ratio and NK cytotoxicity, and the expression of monocyte chemoattractant protein-1 was also increased, whilst the expression levels of NF- $\kappa \mathrm{B}$ and muscle RING finger protein-1 were decreased. Evidently the tumor masses and losses of carcass and/or gastrocnemius muscle were significantly decreased. In addition, data from a meta-analysis regarding CHM as an adjunctive therapy for advanced non-small cell lung cancer reported that CHM combined with conventional chemotherapy markedly reduced the nausea and vomiting at toxicity grade of III-IV and prevented the decline of hemoglobin and platelets in patients under chemotherapy at a toxicity grade of I-V (71). A systematic review suggested that CHM, as an adjuvant therapy, can reduce chemotherapy toxicity, prolong survival time, enhance immediate tumor response and improve the Karnofsky performance status in advanced non-small cell lung cancer patients. The review concluded that Liu-Jun-Zi-Tang, PHY906, coumarin and aescine were capable of improving the postoperation symptoms, such as fatigue, pain, appetite, diarrhea, nausea, vomiting and lymphedema in clinical trials (9). Additionally, ginseng, Huangqi, Ban Zhi Lian, TJ-48, Huachansu injection, Shenqi fuzheng injection and Kanglaite injection in combination with chemo- or radiotherapy were capable of enhancing the efficacy and diminishing the side effects and complications caused by chemo- and radiotherapy, including gastrointestinal side 


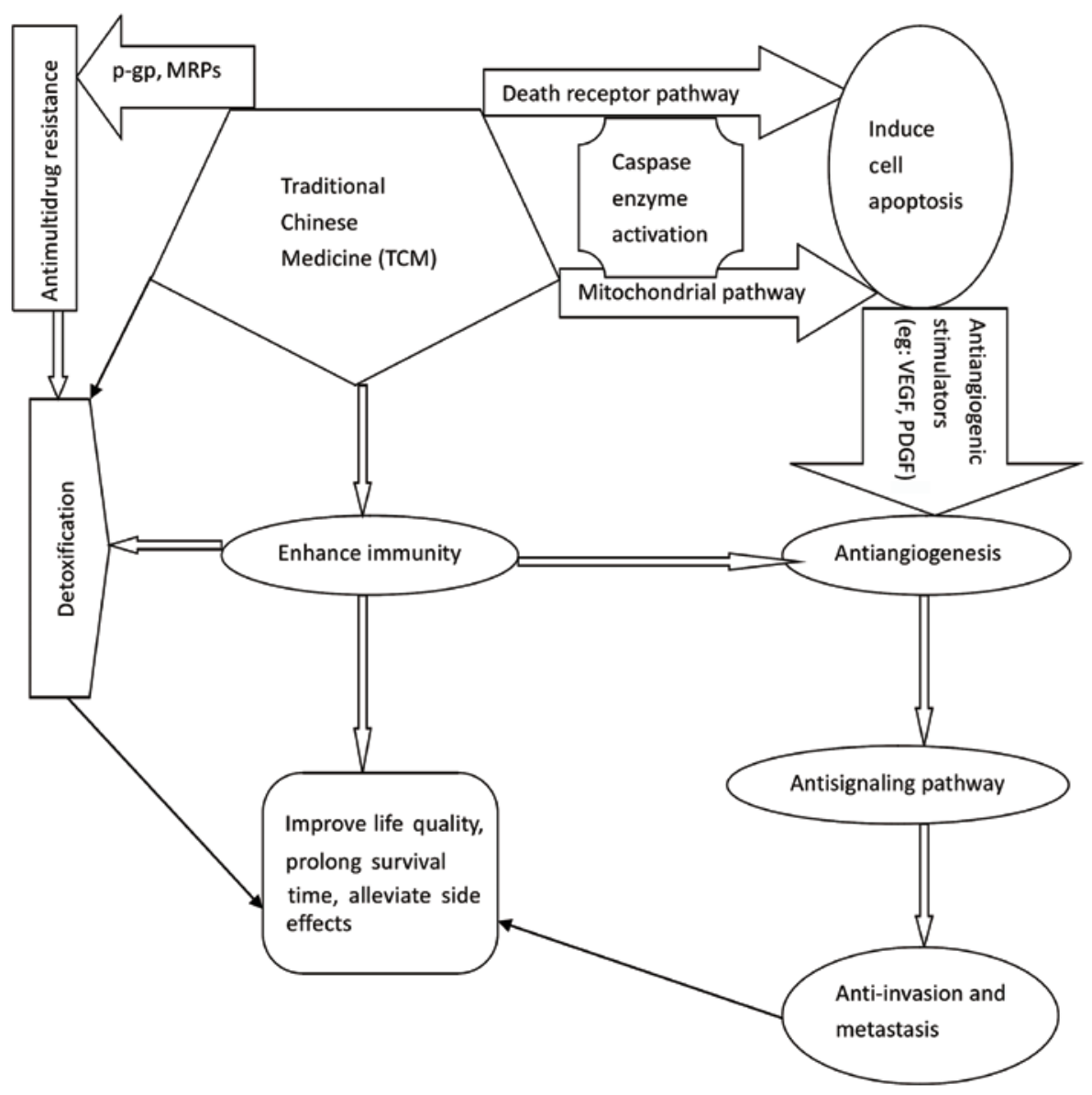

Figure 1. Association of the anticancer mode of action with TCM in the tumor microenvironment. TCM anticancer activity involves targeting various steps (cell proliferation, angiogenesis, cell signaling pathways, invasion, metastasis and multidrug resistance) to inhibit tumor development, reduce the toxicity of other therapies to tumors in the microenvironments, and accordingly, to improve the quality of life for patients. TCM, traditional Chinese medicine; MRP, multidrug resistance protein; VEGF, vascular endothelial growth factor; PDGF, platelet-derived growth factor; P-gp, P-glycoprotein.

effects, liver function damage and even bone marrow suppression.

\section{Conclusion and future perspectives}

Cancer is a multifactorial and multistep disease that can change the tumor microenvironment, leading to alterations in tumor metabolism. The circulation of energy regarding glucose, lipid and amino acid metabolism and the formed metabolites (7) (such as lipids, amino acids, nucleotides, certain inflammatory cytokines and HIF) affected the cancer cell proliferation, autophagy, survival and invasion/metastasis and cellular signaling by blocking their required nutrients, their associated pathways (72) and inhibiting the activity of the regulated immune cells (73). However, the above evidence has proved that TCM has comprehensive advantages in treating cancer with regards to these aspects, which also presented a close association with each other (Fig. 1). Therefore, it may be advantageous to identify and develop novel natural compounds, active ingredients, single herbs and combination formulas or prescriptions in TCM with its multitargets and multisignaling pathways on the tumor, without significant toxicity compared to single utilization with other therapies. Even though the theory of TCM in treating cancer is in the early stages, in recent years, with the enhanced knowledge of translational science and molecular biology, herbs and combination formulas have been reported to treat cancer as an effective therapy in preclinical or clinical trials. The database regarding the pharmacological activities and mechanisms of natural compounds revealed that tumors carried diverse driver gene mutations and crosstalked with different intracellular signals in the tumor microenvironment, leading to carcinogenesis (74). Therefore, it is imperative to explore the mechanism of TCM at the molecular levels to clarify the role of more TCM in curing disease, and additionally, to make a scientific foundation for internationalization of TCM. Based on the main principles of TCM regarding dialectic approaches, individualization and patients with different syndromes require treatment with different approaches. Therefore, TCM with regards to treating different syndromes in various individuals requires clarification. In recent years, there have been numerous studies of the Chinese medicinal compound monomer in the field of medical science, however, there are various components in the herbal medicines, and whether there is an interaction of the functional impacts between themselves by one approach or several ways remains to be elucidated. Therefore, further validation of the biodiversity of the herbal medicines in preclinical or clinical trials, either alone or in combination with existing therapy, is required to benefit more patients with cancer. 


\section{References}

1. Smith RA, Manassaram-Baptiste D, Brooks D, Doroshenk M, Fedewa S, Saslow D, Brawley OW and Wender R: Cancer screening in the United States, 2015: A review of current American cancer society guidelines and current issues in cancer screening. CA Cancer J Clin 65: 30-54, 2015.

2. Bray F, Jemal A, Grey N, Ferlay J and Forman D: Global cancer transitions according to the Human Development Index (2008-2030): A population-based study. Lancet Oncol 13: 790-801, 2012.

3. Adams C, Grey N, Magrath I, Miller A and Torode J: The World Cancer Declaration: Is the world catching up? Lancet Oncol 11: 1018-1020, 2010.

4. Bailly C: Ready for a comeback of natural products in oncology. Biochem Pharmacol 77: 1447-1457, 2009.

5. Li X, Yang G, Li X, Zhang Y, Yang J, Chang J, Sun X, Zhou X, Guo Y, Xu Y, et al: Traditional Chinese medicine in cancer care: A review of controlled clinical studies published in chinese. PLoS One 8: e60338, 2013.

6. Sen T and Samanta SK: Medicinal plants, human health and biodiversity: A broad review. Adv Biochem Eng Biotechnol 147: 59-110, 2015.

7. Casey SC, Amedei A, Aquilano K, Azmi AS, Benencia F, Bhakta D, Bilsland AE, Boosani CS, Chen S, Ciriolo MR, et al: Cancer prevention and therapy through the modulation of the tumor microenvironment. Semin Cancer Biol Apr 10, 2015 (Epub ahead of print) doi: 10.1016/j.semcancer.2015.02.007.

8. Aggarwal BB and Shishodia S: Molecular targets of dietary agents for prevention and therapy of cancer. Biochem Pharmacol 71: $1397-1421,2006$

9. Qi F, Zhao L, Zhou A, Zhang B, Li A, Wang Z and Han J: The advantages of using traditional Chinese medicine as an adjunctive therapy in the whole course of cancer treatment instead of only terminal stage of cancer. Biosci Trends 9: 16-34, 2015.

10. Jenkins VK, Timmons AK and McCall K: Diversity of cell death pathways: Insight from the fly ovary. Trends Cell Biol 23 : 567-574, 2013

11. Elmore S: Apoptosis: A review of programmed cell death Toxicol Pathol 35: 495-516, 2007.

12. Boatright KM and Salvesen GS: Mechanisms of caspase activation. Curr Opin Cell Biol 15: 725-731, 2003.

13. Romero A, Novoa B and Figueras A: The complexity of apoptotic cell death in mollusks: An update. Fish Shellfish Immunol 46: 79-87, 2015.

14. Hengartner MO. The biochemistry of apoptosis. Nature 12: $770-776,2000$

15. Hensley P, Mishra M and Kyprianou N: Targeting caspases in cancer therapeutics. Biol Chem 394: 831-843, 2013.

16. de Poot SA, Lai KW, van der Wal L, Plasman K, Van Damme P, Porter AC, Gevaert K and Bovenschen N: Granzyme M targets topoisomerase II alpha to trigger cell cycle arrest and caspase-dependent apoptosis. Cell Death Differ 21: 416-426, 2014

17. Chuang CH, Wang WJ, Li CF, Ko CY, Chou YH, Chuu CP, Cheng TL and Wang JM: The combination of the prodrugs perforin-CEBPD and perforin-granzyme B efficiently enhances the activation of caspase signaling and kills prostate cancer. Cell Death Dis 5: e1220, 2014.

18. Lin J, Chen Y, Wei L, Chen X, Xu W, Hong Z, Sferra TJ and Peng J: Hedyotis Diffusa Willd extract induces apoptosis via activation of the mitochondrion-dependent pathway in human colon carcinoma cells. Int J Oncol 37: 1331-1338, 2010.

19. Ling Q, Xu X, Wei X, Wang W, Zhou B, Wang B and Zheng S: Oxymatrine induces human pancreatic cancer PANC-1 cells apoptosis via regulating expression of Bcl-2 and IAP families, and releasing of cytochrome c. J Exp Clin Cancer Res 30: 66, 2011.

20. Guo B, Zhang T, Su J, Wang K and Li X: Oxymatrine targets EGFR(p-Tyr845) and inhibits EGFR-related signaling pathways to suppress the proliferation and invasion of gastric cancer cells. Cancer Chemother Pharmacol 75: 353-363, 2015

21. Hu M, Zhao M, An C, Yang M, Li Q, Zhang Y, Suetsugu A, Tome Y, Yano S, Fu Y, et al: Real-time imaging of apoptosis induction of human breast cancer cells by the traditional Chinese medicinal herb tubeimu. Anticancer Res 32: 2509-2514, 2012.

22. Hsu SC and Chung JG: Anticancer potential of emodin. BioMedicine 2: 108-116, 2012.

23. Zhang YK, Wang YJ, Gupta P and Chen ZS: Multidrug resistance proteins (MRPs) and cancer therapy. AAPS J 17: 802-812, 2015.
24. Wilkens S: Structure and mechanism of ABC transporters. F1000Prime Rep 7: 14, 2015.

25. Szakács G, Paterson JK, Ludwig JA, Booth-Genthe C and Gottesman MM: Targeting multidrug resistance in cancer. Nat Rev Drug Discov 5: 219-234, 2006.

26. Leslie EM, Deeley RG and Cole SP: Multidrug resistance proteins: Role of P-glycoprotein, MRP1, MRP2, and BCRP (ABCG2) in tissue defense. Toxicol Appl Pharmacol 204: 216-237, 2005.

27. Li Y, Fan L, Sun Y, Miao X, Zhang F, Meng J, Han J, Zhang D, Zhang R, Yue Z, et al: Paris saponin VII from Trillium tschonoskii reverses multidrug resistance of adriamycin-resistant MCF-7/ADR cells via P-glycoprotein inhibition and apoptosis augmentation. J Ethnopharmacol 154: 728-734, 2014.

28. Cort A and Ozben T: Natural product modulators to overcome multidrug resistance in cancer. Nutr Cancer 67: 411-423, 2015.

29. Eichhorn T and Efferth T: P-glycoprotein and its inhibition in tumors by phytochemicals derived from Chinese herbs. J Ethnopharmacol 141: 557-570, 2012.

30. Ugocsai K, Varga A, Molnár P, Antus S and Molnár J: Effects of selected flavonoids and carotenoids on drug accumulation and apoptosis induction in multidrug-resistant colon cancer cells expressing MDR1/LRP. In Vivo 19: 433-438, 2005.

31. Nabekura T, Kamiyama S and Kitagawa S: Effects of dietary chemopreventive phytochemicals on P-glycoprotein function. Biochem Biophys Res Commun 327: 866-870, 2005.

32. Xie XH, Zhao H, Hu YY and Gu XD: Germacrone reverses Adriamycin resistance through cell apoptosis in multidrug-resistant breast cancer cells. Exp Ther Med 8: $1611-1615,2014$

33. Hu T, To KK, Wang L, Zhang L, Lu L, Shen J, Chan RL, Li M, Yeung $\mathrm{JH}$ and Cho $\mathrm{CH}$ : Reversal of P-glycoprotein (P-gp) mediated multidrug resistance in colon cancer cells by cryptotanshinone and dihydrotanshinone of Salvia miltiorrhiza. Phytomedicine 21: 1264-1272, 2014.

34. Adams M, Mahringer A, Kunert O, Fricker G, Efferth T and Bauer R: Cytotoxicity and p-glycoprotein modulating effects of quinolones and indoloquinazolines from the Chinese herb Evodia rutaecarpa. Planta Med 73: 1554-1557, 2007.

35. Adams M, Mahringer A, Bauer R, Fricker G and Efferth T: In vitro cytotoxicity and P-glycoprotein modulating effects of geranylated furocoumarins from Tetradium daniellii. Planta Med 73: 1475-1478, 2007

36. Wu JY, Yu ZL, Fong WF and Shi YQ: Chemotherapeutic activities of Carthami Flos and its reversal effect on multidrug resistance in cancer cells. Afr J Tradit Complement Altern Med 10: 36-40, 2013.

37. Gordon MS, Mendelson DS and Kato G: Tumor angiogenesis and novel antiangiogenic strategies. Int J Cancer 126: 1777-1787, 2010.

38. Bikfalvi A, Moenner M, Javerzat S, North S and Hagedorn M: Inhibition of angiogenesis and the angiogenesis/invasion shift. Biochem Soc Trans 39: 1560-1564, 2011.

39. Ziche $\mathrm{M}$ and Morbidelli L: Molecular regulation of tumour angiogenesis by nitric oxide. Eur Cytokine Netw 20: 164-170, 2009.

40. Jinushi M: Immune regulation of therapy-resistant niches: Emerging targets for improving anticancer drug responses. Cancer Metastasis Rev 33: 737-745, 2014.

41. Wang Z, Dabrosin C, Yin X, Fuster MM, Arreola A, Rathmell WK, Generali D, Nagaraju GP, El-Rayes B, Ribatti D, et al: Broad targeting of angiogenesis for cancer prevention and therapy. Semin Cancer Biol: Jan 15, 2015 (Epub ahead of print) doi: 10.1016/j.semcancer.2015.01.001.

42. Cook KM and Figg WD: Angiogenesis inhibitors: Current strategies and future prospects. CA Cancer J Clin 60: 222-243, 2010

43. Lu KV, Chang JP, Parachoniak CA, Pandika MM, Aghi MK, Meyronet D, Isachenko N, Fouse SD, Phillips JJ, Cheresh DA, et al: VEGF inhibits tumor cell invasion and mesenchymal transition through a MET/VEGFR2 complex. Cancer Cell 22: 21-35, 2012.

44. Eikesdal HP and Kalluri R: Drug resistance associated with antiangiogenesis therapy. Semin Cancer Biol 19: 310-317, 2009.

45. Wei L, Lin J, Xu W, Cai Q, Shen A, Hong Z and Peng J: Scutellaria barbata $\mathrm{D}$. Don inhibits tumor angiogenesis via suppression of Hedgehog pathway in a mouse model of colorectal cancer. Int J Mol Sci 13: 9419-9430, 2012.

46. Yan B, Liu L, Zhao Y, Xiu LJ, Sun DZ, Liu X, Lu Y, Shi J, Zhang YC, Li YJ, et al: Xiaotan Sanjie decoction attenuates tumor angiogenesis by manipulating Notch-1-regulated proliferation of gastric cancer stem-like cells. World J Gastroenterol 20: $13105-13118,2014$ 
47. Wang XF, Zhou QM, Du J, Zhang H, Lu YY and Su SB: Baicalin suppresses migration, invasion and metastasis of breast cancer via p38MAPK signaling pathway. Anticancer Agents Med Chem 13: 923-931, 2013

48. Shen A, Lin J, Chen Y, Lin W, Liu L, Hong Z, Sferra TJ and Peng J: Pien Tze Huang inhibits tumor angiogenesis in a mouse model of colorectal cancer via suppression of multiple cellular pathways. Oncol Rep 30: 1701-1706, 2013.

49. Li F, Wang Y, Wang X, Li J, Cui H and Niu M: Ganoderic acids suppress growth and angiogenesis by modulating the NF- $\kappa \mathrm{B}$ signaling pathway in breast cancer cells. Int J Clin Pharmacol Ther 50: 712-721, 2012.

50. Wen-Sheng W: ERK signaling pathway is involved in p15INK4b/p16INK4a expression and HepG2 growth inhibition triggered by TPA and Saikosaponin a. Oncogene 22: 955-963, 2003

51. Lee Y-C, Cheng TH, Lee JS, Chen JH, Liao YC, Fong Y, Wu CH and Shih YW: Nobiletin, a citrus flavonoid, suppresses invasion and migration involving FAK/PI3K/Akt and small GTPase signals in human gastric adenocarcinoma AGS cells. Mol Cell Biochem 347: 103-115, 2011.

52. Wang N, Feng Y, Cheung F, Wang X, Zhang Z and Feng Y: A Chinese medicine formula Gegen Qinlian decoction suppresses expansion of human renal carcinoma with inhibition of matrix metalloproteinase-2. Integr Cancer Ther 14: 75-85, 2015.

53. Spurrell EL and Lockley M: Adaptive immunity in cancer immunology and therapeutics. Ecancermedicalscience 8: 441, 2014

54. Xiao W, Wu K, Yin M, Han S, Ding Y, Qiao A, Lu G, Deng B Bo P and Gong W: Wogonin inhibits tumor-derived regulatory molecules by suppressing STAT3 signaling to promote tumor immunity. J Immunother 38: 167-84, 2015.

55. Fridman WH, Pagès F, Sautès-Fridman C and Galon J: The immune contexture in human tumours: Impact on clinical outcome. Nat Rev Cancer 12: 298-306, 2012.

56. Huang CF, Lin SS, Liao PH, Young SC and Yang C-C: The immunopharmaceutical effects and mechanisms of herb medicine. Cell Mol Immunol 5: 23-31, 2008.

57. Peng Q, Cai H, Sun X, Li X, Mo Z and Shi J: Alocasia cucullata exhibits strong antitumor effect in vivo by activating antitumor immunity. PLoS One 8: e75328, 2013.

58. Ma YH, Cheng WZ, Gong F, Ma AL, Yu QW, Zhang JY, Hu CY Chen XH and Zhang DQ: Active Chinese mistletoe lectin-55 enhances colon cancer surveillance through regulating innate and adaptive immune responses. World J Gastroenterol 14: 5274-5281, 2008

59. Sun S, Zheng K, Zhao H, Lu C, Liu B, Yu C, Zhang G, Bian Z, Lu A and He X: Regulatory effect of astragalus polysaccharides on intestinal intraepithelial $\gamma \delta \mathrm{T}$ cells of tumor bearing mice. Molecules 19: 15224-15236, 2014

60. Su ZY and Sheen, LY: An evidence-based perspective of Ganoderma Lucidum (Lucid Ganoderma) for cancer patients. In: Evidence-based Anticancer Materia Medica. Cho, W.C.S.(ed) Springer pp245-263, 2011.
61. de la Taille A, Hayek OR, Burchardt M, Burchardt T and Katz AE: Role of herbal compounds (PC-SPES) in hormone-refractoryprostate cancer: Two case reports. J Altern Complement Med 6: 449-451, 2000

62. Hsieh TC and Wu JM: Mechanism of action of herbal supplement PC-SPES: Elucidation of effects of individual herbs of PC-SPES on proliferation and prostate specific gene expression in androgen-dependent LNCaP cells. Int J Oncol 20: 583-588, 2002.

63. Gao Y, Zhou S, Jiang W, Huang M and Dai X: Effects of ganopoly (a Ganoderma lucidum polysaccharide extract) on the immune functions in advanced-stage cancer patients. Immunol Invest 32: 201-215, 2003.

64. Donohoe CL, Ryan AM and Reynolds JV: Cancer cachexia: Mechanisms and clinical implications. Gastroenterol Res Pract 2011: 601434, 2011.

65. Zhang L, Wu C, Zhang Y, Liu F, Wang X, Zhao M and Hoffman RM: Comparison of efficacy and toxicity of traditional Chinese medicine (TCM) herbal mixture LQ and conventional chemotherapy on lung cancer metastasis and survival in mouse models. PLoS One 9: e109814, 2014

66. Zhang L, Wu C, Zhang Y, Liu F, Zhao M, Bouvet $\mathrm{M}$ and Hoffman RM: Efficacy comparison of traditional Chinese medicine LQ versus gemcitabine in a mouse model of pancreatic cancer. J Cell Biochem 114: 2131-2137, 2013.

67. Qi F, Li A, Inagaki Y, Gao J, Li J, Kokudo N, Li XK and Tang W: Chinese herbal medicines as adjuvant treatment during chemoor radio-therapy for cancer. Biosci Trends 4: 297-307, 2010.

68. Zhou QM, Wang XF, Liu XJ, Zhang H, Lu YY, Huang S and Su SB: Curcumin improves MMC-based chemotherapy by simultaneously sensitising cancer cells to MMC and reducing MMC-associated side-effects. Eur J Cancer 47: 2240-2247, 2011.

69. Shakibaei M, Kraehe P, Popper B, Shayan P, Goel A and Buhrmann C: Curcumin potentiates antitumor activity of 5-fluorouracil in a $3 \mathrm{D}$ alginate tumor microenvironment of colorectal cancer. BMC Cancer 15: 250, 2015.

70. Wang H, Chan YL, Li TL and Wu CJ: Improving cachectic symptoms and immune strength of tumour-bearing mice in chemotherapy by a combination of Scutellaria baicalensis and Qing-Shu-Yi-Qi-Tang. Eur J Cancer 48: 1074-1084, 2012.

71. Li SG, Chen HY, Ou-Yang CS, Wang X-X, Yang Z-J, Tong Y and Cho WC: The efficacy of Chinese herbal medicine as an adjunctive therapy for advanced non-small cell lung cancer: A systematic review and meta-analysis. PLoS One 8: e57604, 2013

72. Singh SR, Tan M and Rameshwar P: Cancer metabolism: Targeting metabolic pathways in cancer therapy. Cancer Lett 356 (2 Pt A): 147-148, 2015.

73. Nowarski R, Gagliani N, Huber S and Flavell RA: Innate immune cells in inflammation and cancer. Cancer Immunol Res 1: 77-84, 2013.

74. Nobili S, Lippi D, Witort E, Donnini M, Bausi L, Mini E and Capaccioli S: Natural compounds for cancer treatment and prevention. Pharmacol Res 59: 365-378, 2009. 